Pacific

Journal of

Mathematics

\title{
WILLMORE-CHEN TUBES ON HOMOGENEOUS SPACES IN WARPED PRODUCT SPACES
}

J. Arroyo, M. Barros, and O.J. Garay 


\title{
WILLMORE-CHEN TUBES ON HOMOGENEOUS SPACES IN WARPED PRODUCT SPACES
}

\author{
J. Arroyo, M. Barros, and O.J. Garay
}

\begin{abstract}
We present a new method to obtain Willmore-Chen submanifolds in spaces endowed with warped product metrics and fibers being a given homogeneous space. The main points are: First the invariance of the variational problem of WillmoreChen with respect to the conformal changes in the ambient space metric. Second, the principle of symmetric criticality which allows us to relate the problem with that for generalized elastic curves in the conformal structure on the base.

We obtain some applications of our method, including one, to get a rational one parameter family of Willmore tori in the standard 3-sphere shaped on an associated family of closed free elastic curves in the standard hyperbolic 2-plane.

We also get a 3-dimensional Riemannian manifold which is foliated with leaves being nontrivial Willmore tori.
\end{abstract}

We start by recalling that Willmore-Chen submanifolds in a Riemannian manifold $(M, g)$ are the critical points of the Willmore-Chen functional $\mathcal{W}$, which is defined by

$$
\mathcal{W}(N)=\int_{N}\left(\alpha^{2}+\tau_{e}\right)^{\frac{n}{2}} d v,
$$

where $N$ is an $n$-dimensional compact submanifold immersed in $M$. The mean curvature function and the extrinsic scalar curvature function of $N$ in $(M, g)$ are $\alpha$ and $\tau_{e}$ respectively and $d v$ denotes the volume element relative to the induced metric by $g$ in $N$. This functional is known to be invariant under conformal changes of the metric $g$ of $M$, [9]. When $n=2$, it coincides with the Willmore functional and then its critical points are the Willmore surfaces. Minimal surfaces in the standard sphere (of any dimension) are obvious examples of Willmore surfaces. Articles showing different methods to construct examples of non-minimal Willmore surfaces in spheres are known in the literature (see for example, $[\mathbf{3}, \mathbf{7}, \mathbf{1 0}, \mathbf{1 2}, \mathbf{1 5}]$ ) even in spaces with no constant curvature (see, for example, $[\mathbf{1}, \mathbf{2}]$ ). 
However the first non-trivial known examples of Willmore-Chen submanifolds (of course with dimension greater than two, exactly they have dimension four) were obtained in [6].

The Euler-Lagrange equations associated with the Willmore functional, in spaces of constant curvature, were computed in [16]. Now the nice family of Willmore tori, in the standard 3-sphere, obtained in [15], can also be directly obtained as a family of solutions of these Euler-Lagrange equations. In fact, it is not difficult to see that these equations for Hopf tori reduce to the Euler-Lagrange equations for certain elastic curves on the standard 2-sphere, [11].

Let $\left(M_{1}, g_{1}\right)$ and $\left(M_{2}, g_{2}\right)$ be two Riemannian manifolds with dimensions $n_{1}$ and $n_{2}$ respectively. Given a positive function $f$ on $M_{1}$ (assume inf $f>0$ if $M_{1}$ is not compact) we define on $M_{1} \times M_{2}$ the following metric

$$
g=g_{1}+f^{2} g_{2}
$$

where we omit the pulling back via the canonical projections of $M$ onto its factors. The Riemannian manifold $(M, g)$ is called the warped product of $\left(M_{1}, g_{1}\right)$ (which is called the base) and $\left(M_{2}, g_{2}\right)$ (which is called the fiber) with warping function $f$, (see $[\mathbf{8}, \mathbf{1 3}]$ for details on the subject). The above warped product is simply denoted by $M_{1} \times_{f} M_{2}$.

From now on $(M, g)$ will denote a warped product with fiber $\left(M_{2}, g_{2}\right)$ being a homogeneous space. Let $G$ be the group of isometries of $\left(M_{2}, g_{2}\right)$. The natural action of $G$ on $\left(M_{2}, g_{2}\right)$ is obviously transitive and it can be extended to an action on $(M, g)$ through isometries as follows

$$
M \times G \longrightarrow M ; \quad p . g=\left(p_{1}, p_{2}\right) \cdot g=\left(p_{1}, p_{2} . g\right),
$$

for any $p=\left(p_{1}, p_{2}\right) \in M=M_{1} \times M_{2}$ and $g \in G$. The orbit of this action through $p=\left(p_{1}, p_{2}\right) \in M$ is given by

$$
[p]=\left[\left(p_{1}, p_{2}\right)\right]=\left\{p_{1}\right\} \times M_{2} .
$$

The $\left(n_{2}+1\right)$-dimensional submanifolds in $(M, g)$ which are $G$-invariant are characterized in the following proposition whose proof is evident.

Proposition. Let $N$ be an $\left(n_{2}+1\right)$-dimensional submanifold in $(M, g)$. Then $N$ is $G$-invariant if and only if there exists a curve $\gamma$ in $\left(M_{1}, g_{1}\right)$ such that $N=\gamma \times_{f} M_{2}$.

To understand the following theorem better, we will say that a $r$-generalized elastica in a Riemannian manifold $(P, h)$ is a curve, which is a critical point of the functional 


$$
\mathcal{F}^{r}(\gamma)=\int_{\gamma}\left(\kappa^{2}\right)^{\frac{r+1}{2}} d s
$$

( $\kappa$ denotes the curvature function of $\gamma$ in $(P, h)$ ), defined on the manifold consisting only of regular closed curves or curves which satisfy a given first order boundary data. The Euler-Lagrange equations relative to this variational problem were computed in $[6]$ when $r=3$. However the same computation can be adapted for an arbitrary value of $r$. In particular, when $r=1$, we have the usual concept of free elastica in the sense of $[\mathbf{1 1}]$.

Theorem. Let $(M, g)=M_{1} \times_{f} M_{2}$ and $\left(M_{2}, g_{2}\right)$ a compact homogeneous space of dimension $n_{2}$. Let $\gamma$ be a closed curve immersed in $\left(M_{1}, g_{1}\right)$. The submanifold $N=\gamma \times_{f} M_{2}$ is a Willmore-Chen submanifold in $(M, g)$ if and only if $\gamma$ is a $n_{2}$-generalized elastica in $\left(M_{1}, \frac{1}{f^{2}} g_{1}\right)$.

Proof. Since the Willmore-Chen variational problem is invariant under conformal changes in the ambient space metric, we make the following change of the metric $g=g_{1}+f^{2} g_{2}$ on $M$, just define

$$
\tilde{g}=\frac{1}{f^{2}} g=\frac{1}{f^{2}} g_{1}+g_{2} .
$$

Now the Willmore-Chen submanifolds in $(M, g)$ are those in $(M, \tilde{g})$. Moreover we can take advantage from the Riemannian product structure of $(M, \tilde{g})$.

Let $\mathcal{N}$ be the smooth manifold consisting of the $\left(n_{2}+1\right)$-dimensional compact submanifolds in $(M, \tilde{g})$. The Willmore-Chen functional $\mathcal{W}: \mathcal{N} \longrightarrow$ $R$ is given by (1) and it certainly is invariant under the $G$-action (3) on $(M, g)$, that is $\mathcal{W}(N)=\mathcal{W}(N, g)$ for any $g \in G$. We put $\mathcal{N}_{G}$ to denote the submanifold of $\mathcal{N}$ made up of those submanifolds which are $G$-invariant. According to the Proposition, we have

$$
\mathcal{N}_{G}=\left\{\gamma \times M_{2} / \gamma \text { is a closed curve in } M_{1}\right\} .
$$

We also write $\mathcal{C}$ and $\mathcal{C}_{G}$ to denote the sets of critical points of $\mathcal{W}$ and $\left.\mathcal{W}\right|_{\mathcal{N}_{G}}$ on $\mathcal{N}$ and $\mathcal{N}_{G}$ respectively. The first one is nothing but the set of Willmore-Chen submanifolds.

The principle of symmetric criticality , [14], can be applied here, consequently we have

$$
\mathcal{C} \cap \mathcal{N}_{G}=\mathcal{C}_{G}
$$

Next we obtain $G$-symmetric Willmore-Chen submanifolds by first computing $\mathcal{W}$ on $\mathcal{N}_{G}$, 


$$
\mathcal{W}\left(\gamma \times M_{2}\right)=\int_{\gamma \times M_{2}}\left(\alpha^{2}+\tau_{e}\right)^{\frac{n_{2}+1}{2}} d s d v_{2},
$$

where $d s$ denotes the arc-length of $\gamma$ in $\left(M_{1}, \frac{1}{f^{2}} g_{1}\right)$ and $d v_{2}$ is the volume element of $\left(M_{2}, g_{2}\right)$. Having in mind that $(M, \tilde{g})$ is a Riemannian product, it is not difficult to see that $\tau_{e}$ vanishes identically and $\alpha^{2}=\frac{1}{\left(1+n_{2}\right)^{2}} \kappa^{2}$, where $\kappa$ is the curvature function of $\gamma$ in $\left(M_{1}, \frac{1}{f^{2}} g_{1}\right)$. Therefore (5) can be written as

$$
\mathcal{W}\left(\gamma \times M_{2}\right)=\frac{\operatorname{vol}\left(M_{2}, g_{2}\right)}{\left(1+n_{2}\right)^{1+n_{2}}} \int_{\gamma}\left(\kappa^{2}\right)^{\frac{n_{2}+1}{2}} d s,
$$

which proves the statement.

From now on, we are going to get some applications of the above established result. To get examples of Willmore tori in the standard 3 -sphere we proceed as follows. Let $\Omega$ be the open hemisphere, in the unit 2-sphere, defined in $R^{4}$ by $x_{1}>0$ and $x_{2}=0$. We denote its standard metric of constant curvature 1 , by $g_{o}$. Let $f: \Omega \longrightarrow R$ be the positive function defined as the $x_{1}$ projection. Then $\Sigma=\Omega \times S^{1}$ is the unit 3-sphere where one geodesic was removed. The standard metric $\bar{g}_{o}$ on $\Sigma$ is $g_{o}+f^{2} d t^{2}$, with the obvious meaning. In others words, $\left(\Sigma, \bar{g}_{o}\right)$ is the warped product of $\left(\Omega, g_{o}\right)$ and $\left(S^{1}, d t^{2}\right)$ with warping function $f$.

To better understand the next result, notice that $\Omega$ endowed with the metric $g=\left(1 / f^{2}\right) g_{o}$ is nothing but the standard hyperbolic 2-plane with constant Gaussian curvature -1 . Consequently we can apply the above stated theorem to have:

Corollary 1. Let $\gamma$ be an immersed closed curve in $\Omega$. The torus $T_{\gamma}=$ $\gamma \times S^{1}$ is Willmore in $\Sigma$ if and only if $\gamma$ is a free elastica in the hyperbolic plane $(\Omega, g)$.

The complete classification of free elastica in the standard hyperbolic plane was achieved in [11]. Besides the $m$-fold cover $\eta_{o}^{m}$ of the so called hyperbolic equator $\eta_{o}$ (that is the geodesic circle of radius $\sinh ^{-1}(1)$ in $(\Omega, g)$ ), there exists an integer two parameter family of free elasticae in $(\Omega, g)$, $\left\{\eta^{m, n} / m>1\right.$ and $\left.\frac{1}{2}<\frac{m}{n}<\frac{\sqrt{2}}{2}\right\}$, (see [11] for a geometrical description of this family). Therefore we obtain:

Corollary 2. There exist infinitely many Willmore tori in the standard 3sphere $\Sigma$. This family includes the following two subfamilies:

1) $\left\{T_{\eta_{o}^{m}}=\eta_{o}^{m} \times S^{1} / m\right.$ is a non zero integer, $\}$ and

2) $\left\{T_{\eta^{m, n}}=\eta^{m, n} \times S^{1} / m>1\right.$ and $\left.\frac{1}{2}<\frac{m}{n}<\frac{\sqrt{2}}{2}\right\}$. 
Remark. (1) The reader should compare the Willmore tori just obtained in the standard 3-sphere with those obtained in [15]. Those came from nonfree elasticae in the standard 2-sphere. To be precise, they are Hopf tori shaped on elastic curves in the 2-sphere but the elasticity is constrainted with a Lagrange multiplier $\lambda=4$ (when one regards the Hopf map as a Riemannian submersion). Also, these tori are conformal to those obtained in $[\mathbf{1 2}]$.

(2) We also notice, (see once more [11]) that the total squared curvature of any immersed closed curve in $(\Omega, g)$ satisfies

$$
\int_{\gamma} \kappa^{2}(s) d s \geq 4 \pi
$$

and the equality in (6) holds if and only if $\gamma$ is a circular free elastica in $(\Omega, g)$, that is the hyperbolic equator $\eta_{o}$ which is a geodesic in $\left(\Omega, g_{o}\right)$. Consequently we obtain the following connection with the popular and well known Willmore conjecture

$$
\mathcal{W}\left(T_{\gamma}\right) \geq 2 \pi^{2}
$$

and the equality in (7) holds if and only if $T_{\gamma}$ is the Clifford torus in $\left(\Sigma, \bar{g}_{o}\right)$.

The following construction combined with the above studied argument allow us to give examples of Chen-Willmore submanifolds associated with certain metrics defined on $M=S^{1} \times S^{1} \times P$, where $P$ denotes any compact homogeneous space (with a given metric $d s^{2}$ ) of dimension, say $r$. We start with any metric $g$ on a torus $T=S^{1} \times S^{1}$, as it is known that $g$ is conformal to some flat metric, say $g_{o}$, on $T$. In others words, there exists a positive function $f$ on $T$ such that $g=f^{2} g_{o}$. We consider $M=T \times P$ endowed with the metric $\tilde{g}=g+f^{2} d s^{2}$ and so $\left(M, \frac{1}{f^{2}} \tilde{g}\right)$ is the Riemannian product of the flat torus $\left(T, g_{o}\right)$ and $\left(P, d s^{2}\right)$. Now for any closed free elastica $\gamma$ in the flat torus $\left(T, g_{o}\right)$, the tube $\Upsilon_{\gamma}=\gamma \times P$ is a Willmore-Chen submanifold in $(M, \tilde{g})$.

Let $R^{3}$ be the Euclidean 3 -space endowed with its canonical metric $<,>$. We define the following positive function on $R^{3}, f(p)=1+\frac{1}{2}|p|$, where the point $p \in R^{3}$ is identified with its position vector (relative to some origin in $R^{3}$ ) and $|p|=<p, p>^{\frac{1}{2}}$. We choose any compact, $r$-dimensional, homogeneous space, say $\left(P, d s^{2}\right)$ as above. Then we have:

Corollary 3. There exists a rational one-parameter family of $(r+1)$-dimensional Willmore-Chen submanifolds in $M=R^{3} \times_{f} P$ for any $r$-dimensional compact homogeneous space $\left(P, d s^{2}\right)$.

Proof. In $M$ with the metric $<,>+f^{2} d s^{2}$ we make a conformal change to get $\frac{1}{f^{2}}<,>+d s^{2}$. Then $\left(R^{3}, \frac{1}{f^{2}}<,>\right)$ is nothing but the once punctured 
3 -sphere, $\Sigma$. Now we can use an argument similar to that used in [6], to show the following statement: For any non-zero rational number $q$, there exists a closed helix $\beta_{q}$ in $\Sigma$ which is a $r$-generalized elastica in $\Sigma$. Then we apply the main theorem to conclude that $\beta_{q} \times_{f} P$ is a Willmore-Chen submanifold in $M$.

The last application is dedicated to obtain a 3-dimensional Riemannian manifold which admit a foliation with leaves being non-trivial Willmore tori, we will call it a Willmore foliation and we will say that the manifold is Willmore foliated.

We start from a plane immersed curve $\gamma: I \subset R \longrightarrow R^{2}$ which we assumed to be parametrized by arc-length $s$. Let $M=\gamma \times S^{1} \times S^{1}$ endowed with the metric $g=d s^{2}+f_{1}^{2} d t^{2}+f_{2}^{2} d u^{2}$, where $f_{1}$ and $f_{2}$ are two positive functions on $\gamma$. Certainly $g$ is conformal to the Riemannian product metric $\tilde{g}$ on $M=N \times S^{1}$ given by $\tilde{g}=g_{o}+d u^{2}$, where $g_{o}=d v^{2}+\frac{f_{1}^{2}}{f_{2}^{2}} d t^{2}$ and $N=\gamma \times S^{1}$. Notice that we made a change of parameters in $\gamma$, to be precise we considered $\frac{d s}{d v}=f_{1}(s)$ on $\gamma$. Now a suitable choice of both $f_{1}$ and $f_{2}$ on $\gamma$, allows us to regard $\left(N, g_{o}\right)$ as a surface of revolution in $R^{3}$.

On the other hand the elasticity of the parallels in a surface of revolution was discussed in [5]. The following statement was proved there: Besides the right cylinders (all whose parallels are geodesics and so trivially free elastic curves), the only surfaces of revolution with all the parallels being free elasticae are what were called the trumpet surfaces, such surfaces are free of geodesic parallels, therefore we have, (see [5] for details):

Corollary 4. Let $b$ and $c$ be a couple of real numbers with $c>0$ and $I=$ $\left(-\frac{2}{c}, 0\right) \bigcup\left(0, \frac{2}{c}\right)$. We define the plane curve (a trumpet) $\gamma: I \subset R \longrightarrow R^{2}$ by

$$
\gamma(s)=\left(\frac{c}{4} s^{2}, \frac{s}{2} \sqrt{1-\frac{c^{2}}{4} s^{2}}-\frac{1}{c} \arccos \frac{c s}{2}+b\right) .
$$

Let $f_{1}$ and $f_{2}$ be two positive functions on $I$ with $\frac{f_{1}(s)}{f_{2}(s)}=\frac{c}{4} s^{2}$. Then $M=$ $\gamma \times S^{1} \times S^{1}$ endowed with the metric $g=d s^{2}+f_{1}^{2} d t^{2}+f_{2}^{2} d u^{2}$ admits a nontrivial Willmore foliation with leaves being nontrivial Willmore tori.

Acknowledgement. The authors would like to express their thanks to the referee for his valuable suggestions.

\section{References}

[1] M. Barros, Free elasticae and Willmore tori in warped product spaces, Glasgow Math. J., 40 (1988), 263-270. 
[2] _ Willmore tori in non standard 3-spheres, Math. Proc. Cambridge Phil. Soc., 121 (1997), 321-324.

[3] M. Barros and B.Y. Chen, Stationary 2-type surfaces in a hypersphere, J. Math. Soc. Japan, 39 (1987), 627-648.

[4] M. Barros, A. Ferrández, P. Lucas and M.A. Meroño, Helicoidal filaments in the 3-sphere, Preprint.

[5] M. Barros and O.J. Garay, Free elastic parallels in a surface of revolution, Amer. Math. Monthly, 103 (1996), 149-156.

[6] _ Hopf submanifolds in $S^{7}$ which are Willmore-Chen submanifolds, Math. Z., 228 (1998), 121-129.

[7] M. Barros, O.J. Garay and D.A. Singer, Elasticae with constant slant in the complex projective plane and new examples of Wilmore tori in five spheres, to appear in Tôhoku Math. J.

[8] A.L. Besse, Einstein manifolds, Springer-Verlag, 1987.

[9] B.Y. Chen, Some conformal invariants of submanifolds and their applications, Boll. Un. Mat. Ital., 10 (1974), 380-385.

[10] N. Ejiri, A counter-example for Weiner open question, Indiana Univ. Math. J., 31 (1982), 209-211.

[11] J. Langer and D. Singer, The total squared curvature of closed curves, J. Diff. Geom., 20 (1984), 1-22.

[12] Curves in the hyperbolic plane and mean curvature of tori in 3-space, Bull. London Math. Soc., 16 (1984), 531-534.

[13] B. O’Neill, Semi-Riemannian Geometry, Academic Press, 1983.

[14] R.S. Palais, Critical points theory and minimax principle, Proc. Sympos. Pure Math., 15 (1970), 185-212.

[15] U. Pinkall, Hopf tori in $S^{3}$, Invent. Math., 81 (1985), 379-386.

[16] J.L. Weiner, On a problem of Chen, Willmore et. al., Indiana Univ. Math. J., 27 (1978), 19-35.

Received April 30, 1997 and revised April 27, 1998. This work was partially supported by a DGICYT Grant No. PB97-0784.

Univ. DE Granada

18071 GRANADA

SPAIN

Univ. DE Granada

18071 GRANADA

SPAIN

E-mail address: mbarros@goliat.ugr.es

Universidad del Pais Vasco

BILbAO

SPAIN

E-mail address: mtpgabeo@lg.ehu.es 